\begin{tabular}{|c|c|}
\hline & PORT SAID ENGINEERING RESEARCH JOURNAL \\
Faculty of Engineering - Port Said University \\
Volume (21) No. (2) September 2017 pp. 199:205
\end{tabular}

\title{
Applying Flexibility Spatial Guidelines on an Intensive Care Unit to Increase its sustainability and life expectancy.
}

\author{
Ashraf A. Elmokadem ${ }^{1}$, Osama Abo Elenien ${ }^{2}$ and Mohamed Nour ${ }^{3}$
}

\begin{abstract}
Flexibility in design is one of the sustainability techniques in architecture which applying it shall increase any buildings sustainability and life expectancy. Hospital buildings are the most type of buildings that needs that due to the rapid and frequent changes that happens to the hospital buildings architecture. High specialized departments in specific, needs flexibility more than other departments to cope with the huge load and development in it. ICU department as one of the specialized departments will definitely increase its sustainability if applied flexibility guidelines on it. Flexibility is the ability to change with an ease and without affect any of the other element badly, it has certain guidelines that guides the way to apply flexibility in hospital buildings.
\end{abstract}

\section{INTRODUCTION}

Hospitals are one of the most changing buildings among all other types of buildings. It changes usually due to the rapid and continuous developments in medical equipment as well as the codes and standards related to hospital spaces designs. Intensive care units are one of the most changing departments inside any building of a hospital and this is because the fast pace of equipment developments and the huge load on such a department on a basis of 24 hours a day a 7 days a week.

The ICU department can be categorized in the inpatient care type of space from the other 5 major types of spaces which are: inpatient care, ambulatory care, diagnostic and treatment functions, support services and public spaces. The inpatient care types of space which represented here in the ICU department frequently needs to be changed or renovated on a several waves each can varies for a range of years of $5-30$ year per wave.

Accordingly, there is a huge need to extend these waves as much as possible and increase the life time expectancy as well as increase sustainability for both: departments and hospitals. Applying the new flexibility concept in such a department shall increase its's sustainability and life expectancy.

Flexibility is a new technique to increase buildings sustainability and it's very suitable for hospital buildings specifically. Flexibility concept means that the space is suitable for affording any changes that could happen in the future with time and cost effective manner which means for sure lasting for longer periods.

\footnotetext{
${ }^{1}$ Professor and Head of Architectural engineering and urban planning, faculty of engineering, Port Said University.

Email: elmokadem1@gmail.com

${ }^{2}$ Associate Professor at Architectural engineering and urban planning,

Faculty of engineering, Port Said University.

Email: aboeinen@yahoo.com

${ }^{3}$ Architect at Gulf Medical Company LTD., Jeddah, Saudi Arabia.

Email: Architect.mohamed.nour@gmail.com
}

Flexibility has several techniques, levels and guidelines that can be achieved with and this research should mention this type of information and explain how to apply these guidelines on a live example (King Fahd general Hospital - Jeddah - Saudi Arabia).

\section{AN APPROACH TO INTENSIVE CARE UNIT (ICU).}

\subsection{Department Definition.}

The Medical / Surgical Intensive Care Unit (ICU) is the area for the critically ill who require the highest level of care and continuous physiologic monitoring. The units can be separated into a Medica1 ICU and a surgical ICU. Once the patient's condition is stable they will be transferred to an acute ward or other specialist units for further treatment.

\subsection{Department Components.}

Accessibility is allowed only in specific periods, parking on-street spaces not allowed, only services vehicles can access. The layout shall take into account the functional relationships between bed areas \& utilities, and staff areas $\&$ bed areas. The layout shall also achieve a balance between providing privacy for patients and unobstructed observation. 

The staff base is usually located in the middle of the unit with the patient rooms surrounding it; this allows staff to see patients at all times and to reach them quickly in case of emergency. All support functions such as medication room, clean and dirty utilities, storage ... etc. shall be easily accessible from the nurse station and bed area. [1]

\section{AN APPRAOCH TO FLEXIBILITY CONCEPT.}

\subsection{Flexibility Definition.}

\subsubsection{Language definition.}

The English colloquial usage of the word "flexibility" is: Susceptibility of modification or alteration; capacity for ready adaptation to various purposes or conditions. (Oxford English Dictionary Online, 2009). [2]

\subsubsection{Technical Meaning.}

\section{Flexibility can mean:}

- Capacity for change: change the size or use of spaces, change its capacity, function, or performance.

- Being Fit: the ability for the building to remain fit for purpose or reduced in mismatches between the building and its users.

- Value: maximizing its productive use, to fit both the context of a system's use and its stakeholders' desires at minimum cost.

- Time: Time is presented in two ways throughout the definitions. First to indicate the speed of change; quick transformations, respond readily; and secondly, to indicate through life changes; future changes, in the long term, or extension of use.

The capacity of a building to accommodate effectively the evolving demands of its context, thus maximizing value through life'. [3]

\subsection{Flexibility Benefits.}

Building technologies and designs that enable flexibility have been identified as bringing a number of benefits.

Flexibility plays a major role to improve the building's sustainable attributes. The possibility of lasting through time while the building spaces and components continue to change, generates many opportunities including each one of the pillars of sustainable development, flexibility has several benefits at the levels of:

- Individual level:

- Good interactivity with the building and other users.

- Flexible design allows users to define the environment that suits their needs hence to achieve person environment congruence. Person- Environment Congruence is a vital concept that support sustainability. [4]
- Socially: flexibility allows to satisfy continuously the common and individual needs of people and support their intervention and interaction between them and with the space.

- Economically:

- Fulfil its function more efficiently,

- Remain longer in service,

- Reduce materials consumption through time,

- It can respond to change faster and at lower cost. That guarantees to keep building viability longer. [5]

- Cost \& time effectiveness. The cost and time of refurbishments is reduced if buildings are designed for flexibility. This is echoed in respect of building services. [6]

- Environmentally:

- Reducing resources and energy consumption.

- Ensures a minimum environment perturbation. [7]

- Longevity and viability.

- Building long tern value.

- Functionally:

- Improve productivity by allowing different functions to take place within an intelligent multi-purpose space. [8]

\subsection{Flexibility Levels.}

Flexibility of healthcare facilities can occur on a range of scales, Flexibility levels are classified as 'adaptability', 'convertibility' and 'expandability'.

\subsubsection{Micro-level Flexibility:}

This is the level of flexibility that is of immediate concern. Micro-level flexibility is a:

$>$ Short-term. It's usually named also as:

- Adaptable flexibility: Spaces can be designed to adapt to multiple uses. An example is a patient room that can be adapted for the purpose of simple procedures such as a line insertion. The different function can be accommodated by simply adapting the space because it has been planned to serve a range of possibilities. Adaptable flexibility is the ability to accommodate changing conditions without any change in the environment. [10]

\subsubsection{Macro-level Flexibility:}

It's the flexibility to expand and convert. It's divide to:

Mid-term: For timeframes of one to five years post-occupancy. [10] And it's named as :

- Convertible flexibility: Another type of flexibility is when, with relatively low effort, time, and/or cost, a space can be converted to another use. Examples of this type of flexibility include a patient room with plumbing, gasses, and electrical systems in the wall for future conversion to critical care. Convertible 
flexibility is the ability to accommodate a change after a simple and/or inexpensive physical alteration. [11]

$>$ Long-term: Over a period of years, as annual volumes increase beyond initial projections and design capacity, a given area can efficiently expand internally into a space previously designated for another use. [12] It's also named as:

- Expandable flexibility: can be summarized as the ability to expand or contract a space to accommodate a particular function, as in future expansions. [13]

\section{FUNCTIONAL FLEXIBILITY GUIELINES.}

\subsection{Multi-functionality:}

Multi-functionality refers to physical components of a space, which allow it to be used in different ways and for a set of known functions. [14]

Planning multi-use or shared facility components enables a healthcare organization to use their space efficiently and balance workload peaks and valleys. Examples of multi-use spaces include:

a) Acuity-adaptable patient room:

Its rooms that can be adapted for most levels of acuity by altering staffing levels and equipment; [15] The underlying assumption of the acuity adaptable room concept is that a room is capable of supporting the complete range of care required for the patient population it is intended to serve.

This assumption demands a larger overall room than the traditional standards; there must be sufficient clinical space to support critical care equipment, staff, and procedure. [16]

\subsection{Trans-functionality:}

Trans-functionality is made of physical components that support the creation of new undetermined and unpredictable functions according to the free users' experience and consumption of the space. The design of such a space is 'open in hand' and emphasizes the dynamic of interaction rather than the static feature of space. [17]

This includes space that can be easily adapted for a different functional use by switching out equipment, adding a second bed, or reassigning offices and workstations to another department.

\subsection{Unbundled spaces:}

Unbundling selected services rather than embedding everything into the hospital structure: cannot only reduce an organization's initial capital investment, but can facilitate future space reallocation, contraction, and expansion, as workloads, staffing, and operational processes change over time. [18]

\subsection{Standardized space:}

Standardized space or space standardization can be described as a controlled space that many aspects are entirely defined. Features of a standardized space is described as: ergonomics specifications; modular units; standardizing room sizes; creating similar room patterns; and modular detailing. Within a standardized room: size; shape; layout; size and orientation of windows; location of doors; direction of openings; location of toilets; and the amount of treatment space required for staff to use their medical equipment and deliver healthcare services are all a time specified.

Standardization of the internal design of specialized departments would ensure higher quality. The availability of template designs for various departments is now promoted by some health care providers. These carefully studied templates would provide a proper framework for local designers to follow. The guidelines must provide a general framework that allows for marginal changes related to local needs. [19] Examples of standardized spaces include:

\section{a) Universal patient room:}

Universal patient room is a Standardized, same-handed rooms with a Standardization of location of materials, Constant entry point. It's a standardized unit where patient rooms as well as the support core are standardized. [20]

\subsection{Modularity:}

Modular Construction is a resource efficient, off-site delivery method to construct code-compliant buildings in a quality-controlled setting. Modular construction by nature is material and resource efficient. [21]

Modularity is used also to facilitate reconfiguration, subdivision and easy rearrangement of spaces through modular walls that guarantees evolution of the building through time according to the needs. [22]

\subsection{General Guidelines:}

- Optimize the space and its utilization: if needed to expand, enhance the space density by multiplying the activities places without expending its topological dimension. Take advantage of every millimeter of the space in height and area.

- Include buffer zones: allowing to absorb the overflowing caused by the frequent change of close spaces and to avoid any encroach on the other spaces. The buffer spaces should have their own function which can change according to the needs. For example adding sub-waiting area in the middle of clinics area

- Design internal circulations routes as part of an overall architectural concept: They should be alive, animated, and interactive and should be able to host many activities. Avoid narrow and dead circulations of which the only function is moving from a place to another. And provide Open-ended corridors to allow buildings to expand in one or more directions. [23] 
- Provide more than the minimum spatial areas and floor heights: to facilitate space adaptation to others functions and conditions.

- Spread out the design and construction of the building over different phases: in order to better meet the progressive change and new needs.

- Integrate the building into the surrounding environment: Design the building as intertwined spaces with the immediate natural and built environment.

- Include mobility: install partitions and furniture that are light, mobile, demountable, reusable and recyclable.

- Use Pod facility design: Consists of a small number of rooms clustered around a central core area, which allows for sterile distribution of supplies. Each suite might contain three, four, or five pods, and the suite is accessed by a peripheral corridor surrounding the entire suite. The design provides for isolation and is highly flexible in terms of future expansion. [24]

- Design spaces that are fluid and continuous and think carefully of the design of storage and its location: "'Hot spots' such as intensive care units are as much as possible surrounded by 'soft spaces' such as waiting areas, administration, and ancillary spaces." Banding clinical spaces on the perimeter of the building enables more flexible management of rooms. The mixing of soft spaces (e.g. storage) with hard creates buffers to allow for expansion over time. [25]

- Excess space programmed into each unit: 'Shell space' is space constructed to meet future needs; it is space enclosed by an exterior building shell, but otherwise unfinished inside. [26]

\section{APPLYING FLEXIBILITY GUIDELINES ON AN ICU DEPARTMENT.}

\subsection{Project Information.}

\footnotetext{
- Architects:

- Location:

- Owner:

- Number of beds:

- Floor area:

- Block type: buildings.

- Population served:

- Level of care:

- Area served:

- Medical specialty:

- Project background:

Gulf Medical.

Jeddah, KSA.

MOH. (Governorate).

36 beds.

2.700 square meters.

Tower on podium

ICU Department at King Fahd general hospital in Jeddah has been renovated \& redesigned at 2016 by the researcher along with gulf medical company. The new design has put into consideration to achieve practical flexibility recommendations as well as achieving operational functionality, healing environment, modular techniques and high treatment quality.

2 million person.

Tertiary care services.

Central area services.

General hospital.
}

\subsection{Project Problem.}

The project had a problem of the frequent renovations that happens every 5 years average due to the heavy load on the hospital as it's one of the biggest general hospital in Jeddah city in Saudi Arabia. Most of these renovations resulted in a long stoppage time for the department working times as the renovation process takes a lot of time each time they renovate it because of the lack of flexibility in the department which resulted in the need to reallocate some rooms each time they start renovate it.

\subsection{Project objective}

The project main objective is to design and implement the department in a way that allows for further future renovations processes with an ease and without the need to fully stop the department operation until the finish of the operation. The objective also is to make the rooms itself flexible enough to accommodate any change of function needed later with a simple and easy modifications. All that with keeping in the mind the main components and important regulations that is linked to the ICU departments design so that it assure that applying these flexibility technique will not affect negatively on the quality of operation of the department. As an opposite, it should affect positively in most of cases.

\subsection{Proposal explanation.}

The proposed solution by the researcher has included the following points:

- Multi-functional rooms such as meeting room and staff lounges and using acuity adaptable patient rooms as well.

- Designing the rooms big enough and with the maximum pre installation requirements in order to support Trans-functionality.

- Standardized rooms with similar design for bed location, door and any other facility.

- Space optimized by using most of unusable spaces for store rooms such as locating the stretcher bay at the end of the corridor.

- Included mobility through the pendants in the patient rooms and light medical equipment.

- Unbundling the spaces by using staff facilities and store in between the inpatient care areas.

- Using modularity through the plan by modular rooms design and through the walls by using high quality modular walls.

- Buffer zones at the entrances of the unit and at elevator's lobby. Maximum possible spatial areas and floor heights.

- The construction can be separated into phases as each part of the design include inpatient Rooms, staff facilities, entrances and stores as well. 
- A cluster of rooms with a nurse station in front was used 4 times to represent pod facility design.

- Storage locations were located between inpatient rooms and in places that is expected to be replaced or expanded later.

- Circulation routes were highly clear and familiar with the other floors routes.

- The design included more rooms than what was required in space program through the space optimization.

\subsection{Analysis Criteria.}

The analysis of the project for flexibility level will be based on the previous guidelines that was mentioned before in the previous sections. It will be distributed into points, each point should be counted as achieved or not achieved based on the guidelines and type of designs mentioned in each single guideline.

\subsection{Zoning analysis.}

The zoning of the department has changed before applying flexibility guidelines and after as following.

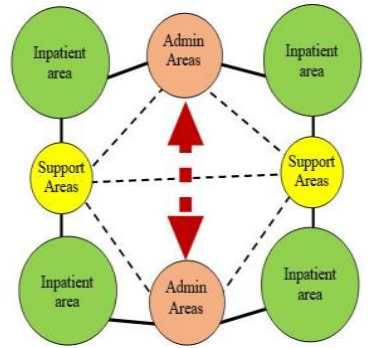

Figure 5.6-2 existing layout zoning diagrame.

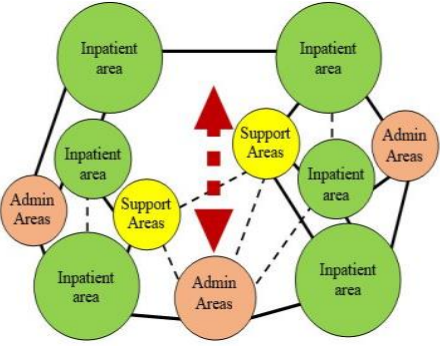

Figure 5.6-1 proposed layout
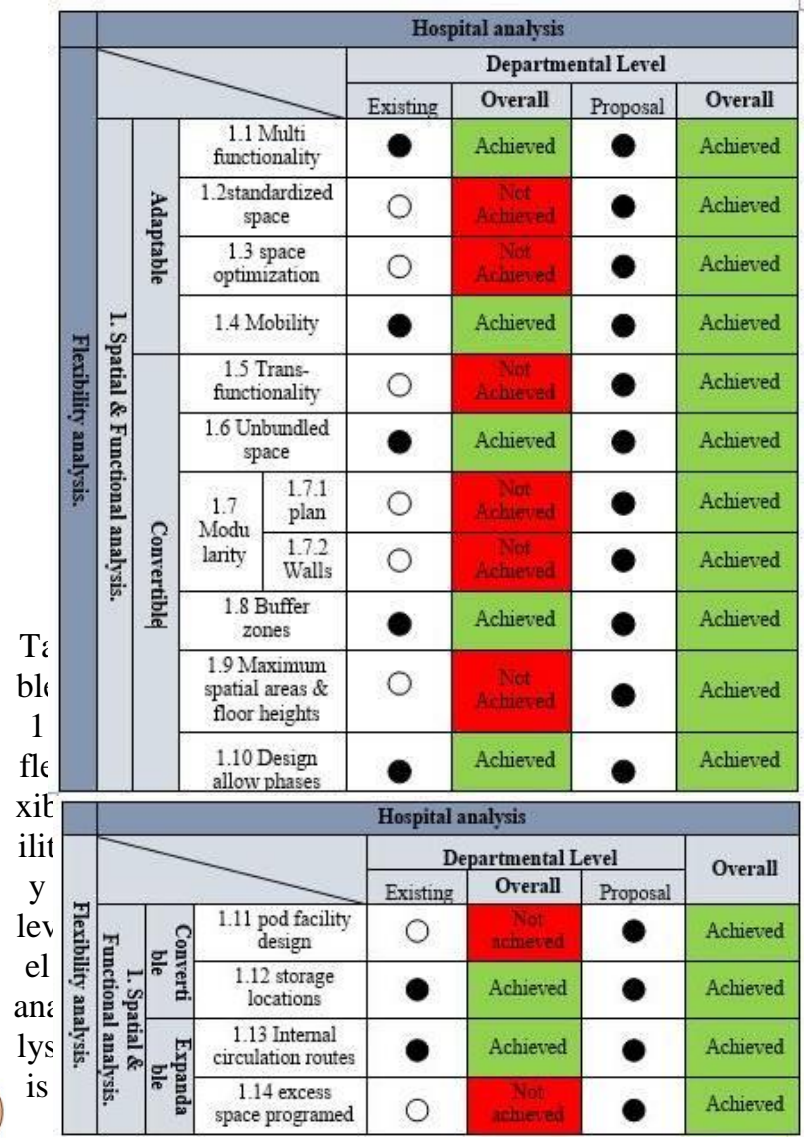

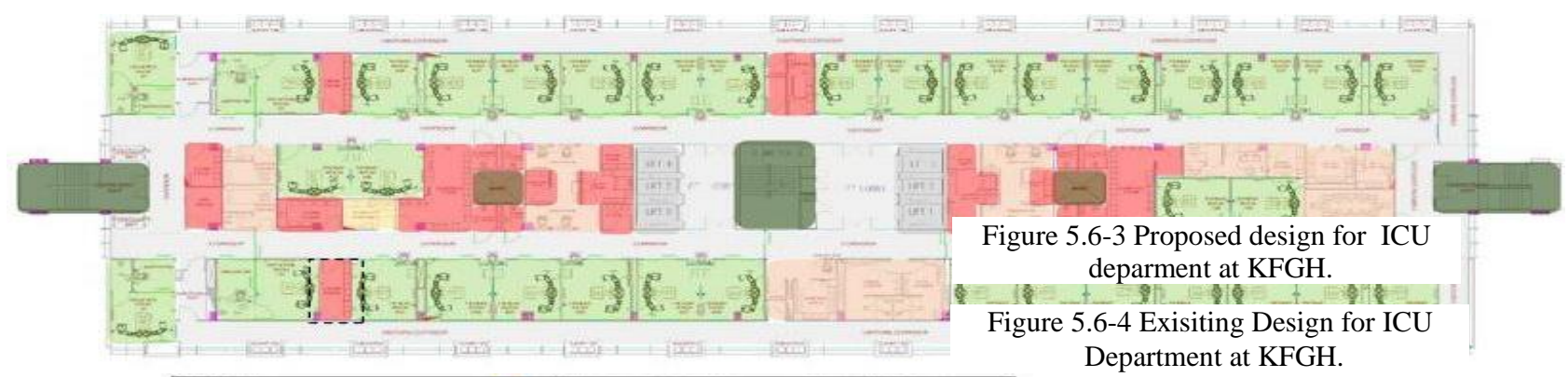

\section{Department at KFGH.}
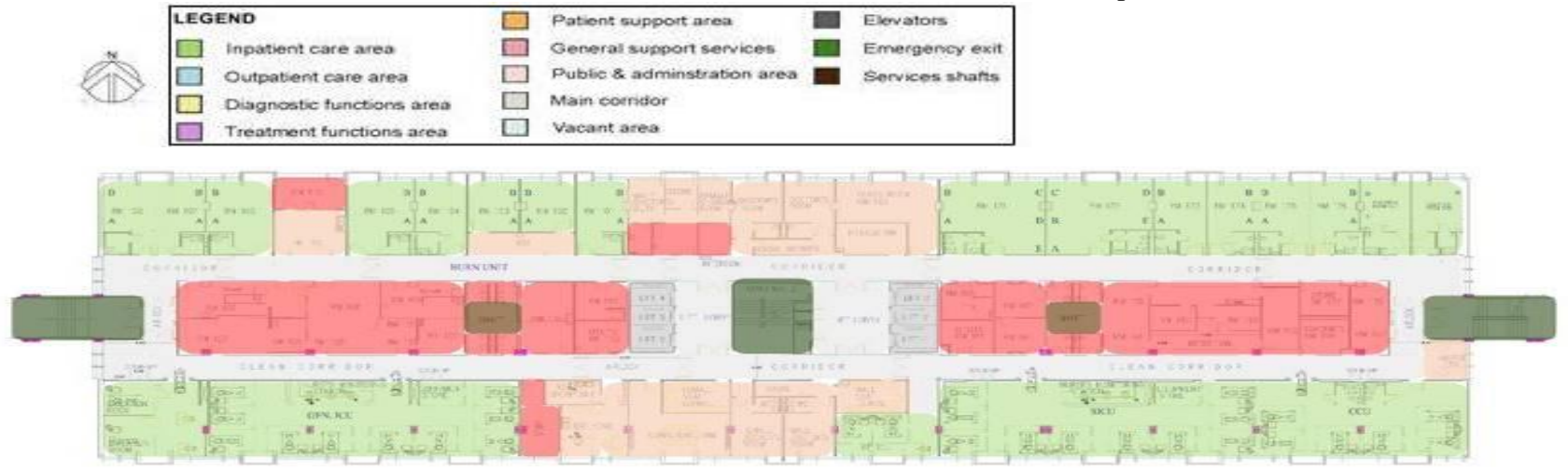


\section{RESULTS.}

- All patient rooms should be single rooms when possible in order facilitate multi-functionality \& trans-functionality and to increase treatment efficiency.

- Use universal patient rooms design whenever possible (standardized rooms \& standardized cluster of rooms with the utilities \& nurse station).

- Use standardized rooms for inpatient departments (standardized space with standardized furniture).

- Increase number of isolation rooms as their main function is isolating patients but they also can be used as a single patient room when needed.

- Use acuity adaptable rooms (the rooms that is equipped \& planned for several functions and its function can be easily changed depending on the need).

- Use maximum spaces possible in all inpatient rooms to accommodate a trans-function to a double patient rooms by adding an additional bed or to an ICU room by adding a charting station.

- Optimize patient wards by adding patient rooms instead of utilities rooms when needed.

- Use modular construction especially in inpatient room's blocks as it's typical all over the building.

- Design more than one corridor \& more than one entrance to allow phases design in inpatient departments.Use multi \& wide corridors with open endings outside patient rooms to connect all patient rooms together. This would help while renovating, separate into phases or expanding.

- The design for inpatient departments specially should allow phases so that the department can operate even without final handover.

- Pod facility design is high applicable in inpatient departments. Using pod facility design in inpatient departments facilitates modularity, standardization, and flexibility during future renovations by removing or adding an additional pods several times.

- Use storages, lounges \& offices around the spaces of ICU in order to facilitate room's expansions.

\section{REFERENCES}

[1] Judith, D. \& Peter, R. (2006). "The art of medical equipment \& furniture planning”. Universal hospital services (UHS). Jordan national library. P.47.

[2] Albostan D. (2009). "Flexibility in multi-residential housing projects". The Graduate school of natural and applied sciences of Middle East technical university. Turkey. Ch.2. P.9-10.

[3] Robert S. \& Toru E. (2010). "What is the meaning of adaptability in the building industry? " Loughborough University. Strategies. P.3-5.

[4] Alaraji K. \& Jusan M. (2012) "Flexible architectural design and user participation”. Conference paper. Teknologi University. Malaysia. Intro. P.2

[5] Nakib F. (2013). "Technological adaptability, an approach toward a flexible and sustainable architecture”. Conference on technology \& sustainability in the built environment. KSU. KSA. VUDD. P.482.

[6] Gosling J., Naim M., Sassi P. \& Lark R. (2008) "Flexible buildings for an adaptable and Sustainable future". 24th Annual ARCOM Conference. Association of Researchers in Construction Management. Review. P.116.

[7] Nakib F. (2013). "Toward an adaptable architecture: Guidelines to integrate adaptability in the building". VUDD. EPAU. Algeria. Intro. P.277.

[8] Ahmed M., Price A. \& Demian P. (2014) “Impact of space flexibility and standardization on healthcare delivery". International journal of applied science and technology. LU. UK. Vol.4. No.4. P.43.

[9] Thomas L. (2010). "Flexibility \& adaptability in hospital design \& construction". DEA 4530. P.18.

[10] Taylor T., Joseph A., Keller A. \& Quan X., (2011). "Designing safety-net clinics for flexibility". California healthcare foundation. The center for health design. CH.V. P.8-9.

[11] JCI, (2008). "Guiding principles for the development of the hospital of the future". Aramark healthcare. pt. V. P.36.

[12] Taylor T., Joseph A., Keller A. \& Quan X., (2011). "Designing safety-net clinics for flexibility". California healthcare foundation. The center for health design. CH.V. P.8-9.

[13] Thomas E., Patti D., Evans J., Cason C. \& Laurie T. (2006). "Inpatient unit design: Defining the design characteristics of a successful adaptable unit". AIA report on university research. School of nursing. University of Texas. Arlington. P. 6.

[14] Ibid 5. P.488.

[15] Hayward C. (2008). "Planning flexible healthcare facilities is no longer optional". Space Med newsletter. P.12.

[16] Taylor T., Joseph A., Keller A. \& Quan X., (2011). "Designing safety-net clinics for flexibility". California healthcare foundation. The center for health design. CH.V. P.14.

[17] Ibid 5. P.488.

[18] Hayward C. (2008). "Planning flexible healthcare facilities is no longer optional". Space Med newsletter. P.23.

[19] Sherif H. (1999). "Hospitals of developing countries: design and construction economics". Journal of architectural engineering. University of Leeds. ASCE. P.78.

[20] Ibid 9. P.21.

[21] The Modular Building Institute, (2010). "Improving construction efficiency \& productivity with modular construction". The voice of commercial modular construction. National academy of science. USA. P.9-12.

[22] Ibid 7. P.281.

[23] Ibid 9. P.42.

[24] Ibid 7.

[25] Ibid 9. P.40. 
[26] Frances Ng. \& Martin R. (2014). "Future-proofing

healthcare facilities". TAHPI. A health planning white paper.

P.5. 